\title{
Metabolic engineering of Escherichia coli for optimized biosynthesis of nicotinamide mononucleotide, a noncanonical redox cofactor
}

\author{
William B. Black', Derek Aspacio ', Danielle Bever ${ }^{1}$, Edward King², Linyue Zhang ${ }^{1}$ and Han Li ${ }^{1 *}$ (0)
}

\begin{abstract}
Background: Noncanonical redox cofactors are emerging as important tools in cell-free biosynthesis to increase the economic viability, to enable exquisite control, and to expand the range of chemistries accessible. However, these noncanonical redox cofactors need to be biologically synthesized to achieve full integration with renewable biomanufacturing processes.

Results: In this work, we engineered Escherichia coli cells to biosynthesize the noncanonical cofactor nicotinamide mononucleotide $\left(\mathrm{NMN}^{+}\right)$, which has been efficiently used in cell-free biosynthesis. First, we developed a growthbased screening platform to identify effective $\mathrm{NMN}^{+}$biosynthetic pathways in E. coli. Second, we explored various pathway combinations and host gene disruption to achieve an intracellular level of $\sim 1.5 \mathrm{mM} \mathrm{NMN}^{+}$, a 130 -fold increase over the cell's basal level, in the best strain, which features a previously uncharacterized nicotinamide phosphoribosyltransferase (NadV) from Ralstonia solanacearum. Last, we revealed mechanisms through which $\mathrm{NMN}^{+}$ accumulation impacts $E$. coli cell fitness, which sheds light on future work aiming to improve the production of this noncanonical redox cofactor.
\end{abstract}

Conclusion: These results further the understanding of effective production and integration of $\mathrm{NMN}^{+}$into E. coli. This may enable the implementation of $\mathrm{NMN}^{+}$-directed biocatalysis without the need for exogenous cofactor supply.

Keywords: Nicotinamide mononucleotide, Noncanonical redox cofactor, Escherichia coli, Metabolic engineering, $\mathrm{NAD}^{+}$biosynthesis, Biomimetic cofactor

\section{Introduction}

In the last decade, cell free biosynthesis has emerged as a prominent tool in the production of renewable chemicals, fuels, and pharmaceuticals [1-3]. Cell-free systems, both purified enzyme-based and crude lysate-based, have unique advantages over whole-cell biotransformation systems. For example, environmental conditions can be varied within a wider range to favor product formation [4]; transportation issues across cell membranes

*Correspondence: hanl5@uci.edu

${ }^{1}$ Departments of Chemical and Biomolecular Engineering, University of California, Irvine, CA, United States

Full list of author information is available at the end of the article are eliminated [5]; toxic compounds can be produced at much higher titers than the cell's tolerance limit [6]. Because components of the biosynthetic pathways can be readily mix-and-matched in a combinatorial fashion, cellfree biosynthesis has also been used as a high-throughput prototyping tool to inform pathway design in whole-cell biosynthesis $[7,8]$.

Cofactors such as nicotinamide adenine dinucleotide (phosphate) $\left(\mathrm{NAD}(\mathrm{P})^{+}\right)$are essential reagents in biosynthesis. In cell-free biosynthesis, cofactors are freed from the life-sustaining roles they play in vivo. Therefore, true opportunities exist to significantly expand the toolkit of cofactors beyond what is offered by Nature to achieve desirable goals in biocatalysis. For example, cheaper 
noncanonical cofactors, such as 3-carbamoyl-1-phenethylpyridin-1-ium chloride $\left(\mathrm{P}^{2} \mathrm{NA}^{+}\right)[9,10]$, have been used in purified enzyme-based redox catalysis to increase economic viability. Noncanonical cofactors with stronger electron-accepting capability, such as 3-acetylpyridine adenine dinucleotide [11, 12], have been used to drive the thermodynamically unfavorable reactions of alcohol oxidation.

We recently developed a cell-free biosynthesis platform surrounding the noncanonical redox cofactor nicotinamide mononucleotide $\left(\mathrm{NMN}^{+}\right)$[13]. $\mathrm{NMN}^{+}$was enzymatically cycled by pairing an engineered glucose dehydrogenase from Bacillus subtilis with a variety of enzymes to reduce activated $\mathrm{C}=\mathrm{C}$ double bonds, activated $\mathrm{C} \equiv \mathrm{C}$ triple bonds, nitro groups, and to supply electrons to a cytochrome $\mathrm{P} 450$. This system demonstrated robust temporal stability over $96 \mathrm{~h}$ and a total turnover number of $\sim 39,000$. Because of its smaller size, $\mathrm{NMN}^{+}$has also been shown to provide a faster mass transfer rate in enzymatic biofuel cells [14].

Compared to other noncanonical cofactors which are made through chemical synthesis [15-17], $\mathrm{NMN}^{+}$is particularly suited for fully renewable biomanufacturing processes because it is accessible through biosynthesis [18-20]. This feature is especially desirable in crude lysate-based cell-free biosynthesis and whole-cell biosynthesis, where $\mathrm{NMN}^{+}$produced in the cells does not need to be purified or exogenously supplied, and it can be directly used for downstream biocatalysis. Importantly, since we demonstrated its successful application in E. coli whole cells to enable orthogonal electron delivery [13], $\mathrm{NMN}^{+}$can potentially be utilized in crude lysate-based biosynthesis to control the flow of reducing power and mitigate side reactions based on the same principles [13, 21].

Although $\mathrm{NMN}^{+}$has been biosynthesized previously in metabolically engineered E. coli $[13,19]$, further improving $\mathrm{NMN}^{+}$production requires more efficient pathways and a better understanding of its metabolism in the host. While previous efforts have primarily used the nicotinamide phosphoribosyltransferases, NadV, to convert nicotinamide to $\mathrm{NMN}^{+}$, only a few NadV homologs have been tested and many other NadV-independent pathways for $\mathrm{NMN}^{+}$biosynthesis remain unexplored. Furthermore, whether and how $\mathrm{NMN}^{+}$accumulation impacts cell physiology remains largely unknown. In this work, we developed a growth-based screening platform to identify pathways for efficient $\mathrm{NMN}^{+}$generation in vivo. This platform was designed by making $\mathrm{NMN}^{+}$an essential precursor in $\mathrm{NAD}^{+}$biosynthesis in engineered E. coli. We used this platform to demonstrate that $\mathrm{NMN}^{+}$ synthetase, NadE* from Francisella tularensis, effectively mediates an additional route for $\mathrm{NMN}^{+}$biosynthesis in E. coli. We also bioprospected for NadV homologs based on comparative genomic data [18], and we tested their ability to produce $\mathrm{NMN}^{+}$in combination with F. tularensis $\mathrm{NadE}^{*}$. The best $\mathrm{NMN}^{+}$producing strain accumulated $\sim 1.5 \mathrm{mM}$ of intracellular $\mathrm{NMN}^{+}$while overexpressing F. tularensis $\mathrm{NadE}^{*}$ and Ralstonia solanacearum NadV simultaneously, as well as harboring a disruption in the gene encoding $\mathrm{NMN}^{+}$amidohydrolase, PncC. Although our current highest $\mathrm{NMN}^{+}$production titer did not cause growth inhibition, we observed inhibitory effect when very high concentrations of $\mathrm{NMN}^{+}$was fed to the cells through a heterologous $\mathrm{NMN}^{+}$transporter. Interestingly, we showed that this inhibitory effect can be alleviated when the transcriptional regulator of $\mathrm{NAD}^{+}$biosynthesis, NadR, was disrupted. Together, these results provide insight for future metabolic engineering efforts aiming to further improve $\mathrm{NMN}^{+}$biosynthesis. Compared to $\mathrm{NAD}(\mathrm{P})^{+}, \mathrm{NMN}^{+}$has been suggested to be less expensive [22]. The development of an efficient $\mathrm{NMN}^{+}$biosynthetic route from even cheaper precursors may further increase the economical viability of $\mathrm{NMN}^{+}$-dependent biotransformation processes.

\section{Results}

\section{Identification of $\mathrm{NMN}^{+}$biosynthetic routes}

In $E$. coli cells, $\mathrm{NMN}^{+}$is only present at a nominal level, $\sim 11.5 \mu \mathrm{M}$ as previously reported [13], as the product of the DNA ligase reaction [18]. On the other hand, $\mathrm{NMN}^{+}$accumulates to higher levels and serves as a main intermediate in $\mathrm{NAD}^{+}$biosynthesis in other organisms $[23,24]$. Here, we sought to systematically investigate the effectiveness of these heterologous $\mathrm{NMN}^{+}$biosynthetic routes in E. coli (Fig. 1).

Three major $\mathrm{NMN}^{+}$biosynthetic pathways exist in Nature (Fig. 1): Pathway 1 produces $\mathrm{NMN}^{+}$from nicotinic acid mononucleotide $\left(\mathrm{NaMN}^{+}\right)$using $\mathrm{NMN}^{+}$ synthetase $\left(\mathrm{NadE}^{*}\right)$, and it was shown to be part of the de novo $\mathrm{NAD}^{+}$biosynthetic pathway in a small group of prokaryotes including $F$. tularensis [24]. Pathway 2 involves phosphorylation of nicotinamide riboside (NR) and functions to salvage NR to ultimately yield $\mathrm{NAD}^{+}$. To establish this pathway, we chose to overexpress the native NR transporter in E. coli, PnuC [25], in conjunction with two different NR kinases, Nrk1 from Saccharomyces cerevisiae [26] and NadR from Salmonella enterica [27]. Pathway 3 uses nicotinamide phosphoribosyltransferase (NadV) to convert nicotinamide (NA) to $\mathrm{NMN}^{+}$; it plays a role in NA salvage in vertebrates and some bacteria. Marinescu and coworkers demonstrated $\mathrm{NMN}^{+}$accumulation in E. coli by heterologously expressing three NadV homologs from Haemophilus ducreyi, Shewanella oneidensis, and Mus musculus while feeding NA [19], and they showed that 


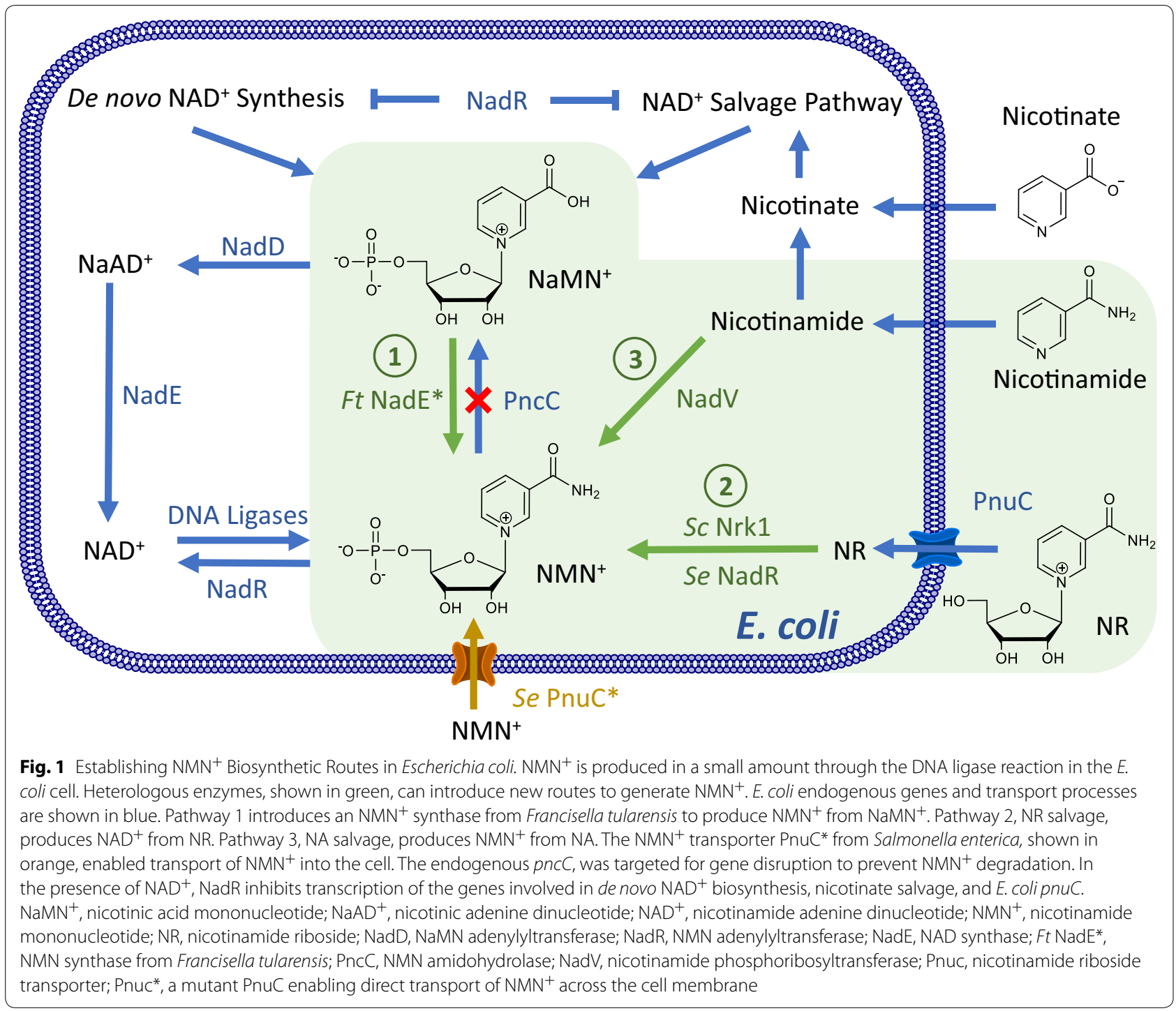

H. ducreyi NadV performed the best [19]. We previously showed that NadV from F. tularensis, which belongs to a different clade in the NadV phylogenetic tree to $H$. ducreyi NadV [18], can also effectively produce $\mathrm{NMN}^{+}$in E. coli [13]. Here, we sought to explore more bacterial NadVs in the same family of $F$. tularensis NadV and compare them with $H$. ducreyi and F. tularensis NadV. Namely, we chose NadV homologs from $R$. solanacearum, Synechocystis sp., and Synechococcus elongatus [18].

All three pathways have unique advantages. While Pathway 3 requires a much cheaper substrate than Pathway 2 (NA versus NR), the latter incorporates ATP hydrolysis as a robust driving force. Since $\mathrm{NaMN}^{+}$is an intermediate in $E$. coli's de novo $\mathrm{NAD}^{+}$biosynthesis and can be efficiently produced from central metabolites (Fig. 1), Pathway 1 has the potential to achieve complete de novo $\mathrm{NMN}^{+}$biosynthesis from simple feed stocks such as glucose.

\section{Evaluating the $\mathrm{NMN}^{+}$biosynthetic pathways in vivo}

We evaluated the three above-mentioned pathways in E. coli using a growth-based screening platform (Figs. 1 and 2). To link $\mathrm{NMN}^{+}$production to cell survival, we employed $E$. coli strain 72 c, which contains a temperature sensitive allele of $n a d D$, an essential gene in $\mathrm{NAD}^{+}$ biosynthesis. As a result, the cells cannot grow at $42{ }^{\circ} \mathrm{C}$ [28] unless $\mathrm{NMN}^{+}$can accumulate inside the cells and be converted to $\mathrm{NAD}^{+}$by E. coli NadR (Figs. 1 and 2). Previous work has also established $\mathrm{NMN}^{+}$-dependent $\mathrm{NAD}^{+}$biosynthesis to rescue $\mathrm{NAD}^{+}$auxotrophy in $E$. coli [29]. 

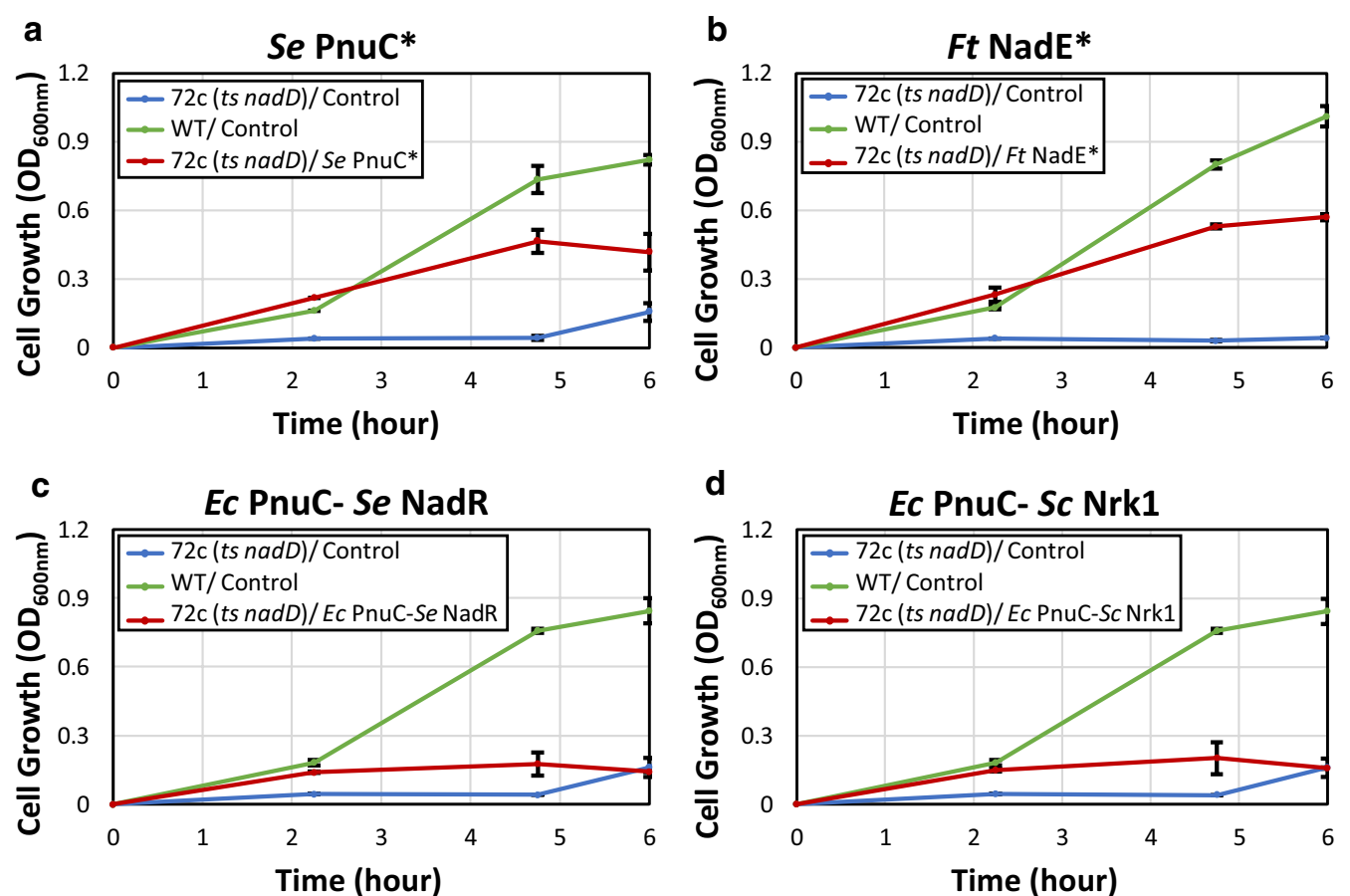

Fig. 2 Identification of efficient NMN ${ }^{+}$production pathways in vivo using a growth-based screening platform. A growth-base screening platform was used to identify pathways which efficiently generated $\mathrm{NMN}^{+}$in vivo. E. coli strain $72 \mathrm{c}$, which contains a temperature sensitive allele of nadD (ts nadD), which exhibits a conditionally lethal phenotype when cultured at $42^{\circ} \mathrm{C}$ because the native $\mathrm{NAD}^{+}$biosynthesis is disrupted. Therefore, the cell must rely on intracellular $\mathrm{NMN}^{+}$to restore $\mathrm{NAD}^{+}$formation and growth. a Direct feeding of $\mathrm{NMN}^{+}$into the growth medium with the overexpression of an $\mathrm{NMN}^{+}$transporter, PnuC* from S. enterica, restored growth to levels near the wild type control, indicating the platform is effective for $\mathrm{NMN}^{+}$production screening. $\mathbf{b}$ Introducing $F$. tularensis $\mathrm{NadE}^{*}$ also restored growth with the supplementation of nicotinamide. $\mathbf{c}$, $\mathbf{d}$ Overexpression of E. coli's native nicotinamide riboside (NR) transporter PnuC, paired with S. enterica NadR (c) or S. cerevisiae Nrk1 (d) while feeding NR failed to efficiently restore growth. Screening was performed in a deep-well 96-well plate containing $1 \mathrm{~mL}$ of LB medium supplemented with $2 \mathrm{~g} / \mathrm{L} \mathrm{D}$-glucose and $200 \mu \mathrm{M}$ of $\mathrm{NMN}^{+}$precursors, if applicable

When $200 \mu \mathrm{M} \mathrm{NMN}{ }^{+}$was directly fed to the cells expressing an $\mathrm{NMN}^{+}$transporter from S. enterica PnuC* [30] (on a multiple-copy plasmid pWB302), growth was restored to levels comparable to wild type cells after $6 \mathrm{~h}$ (Fig. 2a). In contrast, cells carrying a control plasmid (pWB301) could not grow, suggesting the basal level of $\mathrm{NMN}^{+}$in E. coli cells does not cause background issues in this growth-based screening, possibly because $E$. coli NadR has low affinity towards $\mathrm{NMN}^{+}$[31]. These results demonstrate that the screening platform is functioning properly, and cell growth is serving as a readout for intracellular $\mathrm{NMN}^{+}$level. We and others have previously shown that $\mathrm{NMN}^{+}$can enter $E$. coli cells without expressing a heterologous transporter [13, 29]. In this work, the amount of $\mathrm{NMN}^{+}$supplementation can be substantially reduced, suggesting that $S$. enterica PnuC* improves the efficiency of $\mathrm{NMN}^{+}$transportation into $E$. coli cells.

Overexpression of $F$. tularensis NadE* (Fig. 1, Pathway 1 , on the plasmid pWB303) with $200 \mu \mathrm{M}$ nicotinamide supplementation restored growth to a similar level as directly feeding $\mathrm{NMN}^{+}$(Fig. 2b). Nicotinamide can yield the substrate of $\mathrm{NadE}^{*}$, namely $\mathrm{NaMN}^{+}$, through $E$. coli 's native salvage pathway. Interestingly, growth restoration by $F$. tularensis NadE* does not depend on nicotinamide feeding (Additional file 1: Figure $\mathrm{S} 1$ ). $\mathrm{NMN}^{+}$production using S. cerevisiae Nrk1 or S. enterica NadR (Fig. 2, Pathway 2, on the plasmids pWB304 and pWB305) with $200 \mu \mathrm{M}$ NR supplementation was not efficient enough to restore growth (Fig. 2c, d). This may be due to the poor expression of these heterologous kinases in E. coli and their relatively high $K_{\mathrm{M}}$ for NR and ATP [30]. Therefore, we did not proceed with Pathway 2.

Taken together, these results suggest that besides the well-established NadV route (Fig. 1, Pathway 3) [19], the F. tularensis NadE*-dependent pathway (Pathway 1) is also effective in $E$. coli for $\mathrm{NMN}^{+}$biosynthesis.

\section{Bioprospecting NadV homologs and optimizing $\mathrm{NMN}^{+}$ biosynthesis}

After demonstrating $\mathrm{NMN}^{+}$can be effectively generated by overexpressing $F$. tularensis $\mathrm{NadE}^{*}$, we examined the effects of pairing it with different NadV homologs. 
F. tularensis NadE* was overexpressed in a synthetic operon on a multiple-copy plasmid with each of the five NadV candidates as described above (pWB203, pDB101, pDB102, pDB103, pDB104). Cells were fed $1 \mathrm{mM}$ of NA and grown for $4 \mathrm{~h}$ before processing and quantification of intracellular $\mathrm{NMN}^{+}$and $\mathrm{NAD}^{+}$levels via LC-MS analysis as previously described [13]. When using the wild type BW25113 cells as the host, 12 to $51 \mu \mathrm{M}$ of $\mathrm{NMN}^{+}$ was produced through these pathways (Fig. 3). We previously found that low levels of intracellular $\mathrm{NMN}^{+}$could be attributed to $\mathrm{NMN}^{+}$degradation by the $\mathrm{NMN}^{+}$amidohydrolase, PncC [13]. Expression of the $\mathrm{NadE}^{*} / \mathrm{NadV}$ pathways in a $\Delta p n c C$ strain, JW2670-1 significantly increased the intracellular $\mathrm{NMN}^{+}$levels. When the NadV candidates were expressed without $F$. tularensis $\mathrm{NadE}^{*}$ in JW2670-1 (on plasmids pWB303, pWB306, pWB307, pWB308, pWB309), intracellular $\mathrm{NMN}^{+}$levels were lower for all NadVs except $H$. ducreyi (Fig. 3). Notably, expressing $R$. solanacerum and S. elongatus NadVs alone resulted in a significant growth challenge (Additional file 1: Figure S2) and the concomitant diminishing of $\mathrm{NMN}^{+}$production (Fig. 3). However, this growth challenge could be overcome by expressing the $\mathrm{NadV}$ with $F$. tularensis $\mathrm{NadE}^{*}$ (Additional file 1: Figure S2), indicating that $F t \mathrm{NadE}^{*}$ may provide a synergistic benefit to the stability, activity, or expression for some NadV candidates. Future work is needed to pinpoint the molecular mechanism behind this synergy.

Cells expressing $F$. tularensis $\mathrm{NadE}^{*}$ and $R$. solanacearum NadV in the $\Delta p n c C$ strain reached the highest intracellular $\mathrm{NMN}^{+}$level of $\sim 1.5 \mathrm{mM}$, a 130 -fold increase over the cell's basal $\mathrm{NMN}^{+}$level [13], when tested under the same conditions (Fig. 3). Furthermore, the R. solanacearum NadV strain performed better than $F$. tularensis $\mathrm{NadV}$, the NadV we used in our previous work [13], exhibiting a 2.8-fold increase in intracellular $\mathrm{NMN}^{+}$

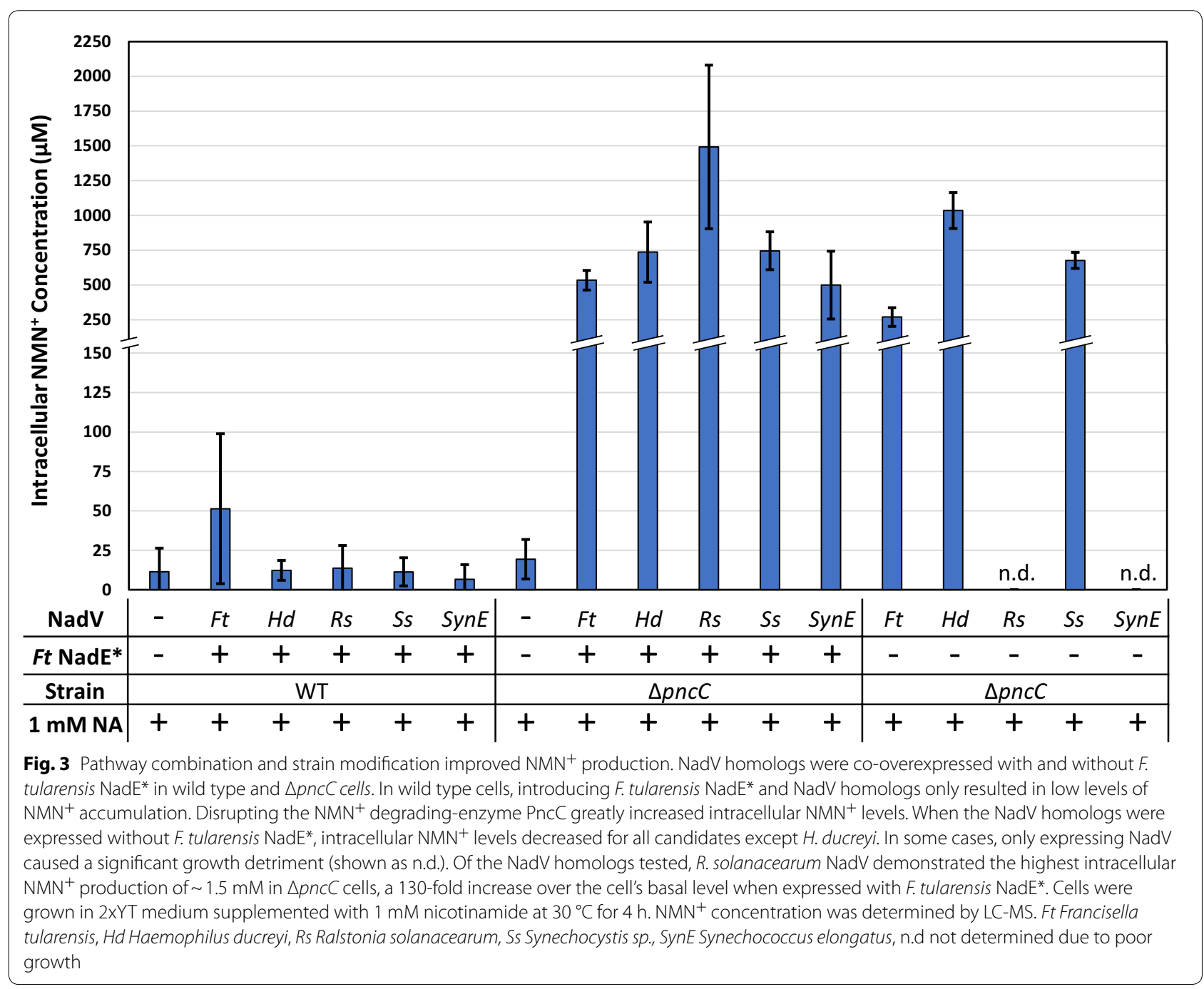


concentration. $R$. solanacearum NadV also performed better than $H$. ducreyi $\mathrm{NadV}$ [19] when paired with $F$. tularensis NadE*.

\section{Investigating the physiological response to $\mathrm{NMN}^{+}$ accumulation}

Even though the de novo $\mathrm{NAD}^{+}$biosynthesis pathway was unmodified, the $\triangle p n c C$ cells overexpressing $F$. tularensis NadE* and NadV homologs had lower intracellular $\mathrm{NAD}^{+}$levels compared to the control strain in which no proteins were overexpressed and intracellular $\mathrm{NMN}^{+}$ level was low (Fig. 3, Additional file 1: S3). This suggests that maintaining intracellular $\mathrm{NMN}^{+}$at millimolar-range concentrations may be detrimental to cellular fitness. Although we observed no growth defects in our current best $\mathrm{NMN}^{+}$producing strain, the potential physiological effects of $\mathrm{NMN}^{+}$accumulation may become a bottleneck for future strain optimization.

Since the effects of $\mathrm{NMN}^{+}$accumulation on E. coli are not well understood, we sought to stress the cells by drastically increasing the intracellular $\mathrm{NMN}^{+}$levels in the $\Delta p n c C$ strain and observe cellular growth. $\mathrm{NMN}^{+}$ concentration was titrated in the medium while expressing the exogenous $\mathrm{NMN}^{+}$transporter S. enterica PnuC** We found that cell growth was inhibited at high $\mathrm{NMN}^{+}$ concentrations (>5 mM) (Fig. 4), which suggests that elevated $\mathrm{NMN}^{+}$level may interfere with physiological processes in E. coli. Although intracellular $\mathrm{NMN}^{+}$levels were not measured in this application, PnuC $C^{*}$-mediated $\mathrm{NMN}^{+}$transport was shown to be active with as low as $200 \mu \mathrm{M}$ of $\mathrm{NMN}^{+}$supplementation to support growth restoration (Fig. 2a). Given the decrease in intracellular $\mathrm{NAD}^{+}$level upon $\mathrm{NMN}^{+}$accumulation (Additional file 1: Figure S3), we hypothesized that $\mathrm{NMN}^{+}$may regulate $\mathrm{NAD}^{+}$biosynthesis, and we sought to examine whether this regulation was mediated by the transcriptional regulator of $\mathrm{NAD}^{+}$biosynthesis, NadR [32]. Interestingly, when NadR was disrupted, the growth inhibition effect of $\mathrm{NMN}^{+}$was significantly alleviated (Fig. 4). These results suggest that NadR may indeed play a role in the physiological response to $\mathrm{NMN}^{+}$accumulation in E. coli. $\mathrm{NAD}^{+}$ has been suggested to allosterically modulate NadR's function [33]. Given NadR's capability to also recognize $\mathrm{NMN}^{+}$[31], further studies are needed to investigate whether $\mathrm{NMN}^{+}$binding induces conformational change in the DNA-binding domain of NadR and modulates its function as a transcriptional regulator.

\section{Discussion}

This work represents the initial steps towards filling some of the fundamental knowledge gaps that remained open in previous work on $\mathrm{NMN}^{+}$biosynthesis in E. coli. The important work by Marinescu and coworkers [19]

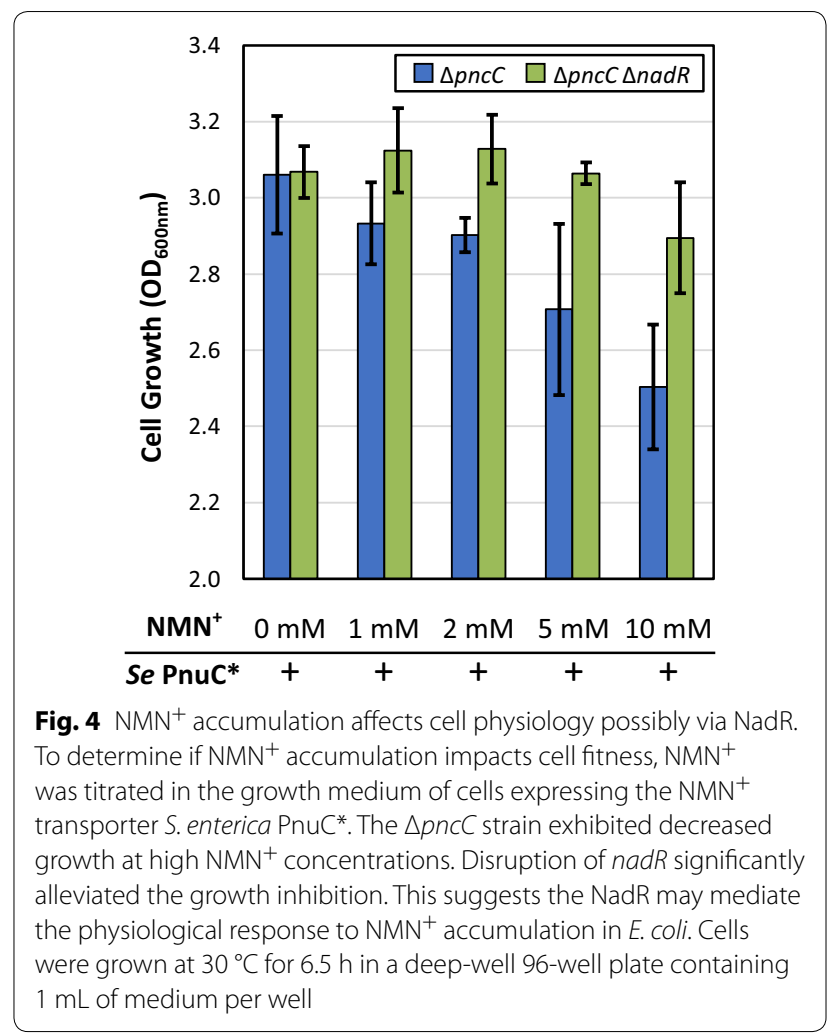

focused on the NadV pathway, but it left many other naturally occurring $\mathrm{NMN}^{+}$biosynthetic routes unexplored. Our previous work [13] sought to simply recapitulate the $\mathrm{NMN}^{+}$metabolism of $F$. tularensis [24] by overexpressing both $\mathrm{NadE}^{*}$ and $\mathrm{NadV}$ from this organism, without dissecting the role of each pathway. Moreover, both efforts did not study the physiological response in $E$. coli to $\mathrm{NMN}^{+}$accumulation. Beside functioning as a cofactor, $\mathrm{NAD}^{+}$is also a universal signaling compound that allosterically controls key enzymes and transcriptional regulators in response to the fluctuating cellular redox state [31, 34-37]. Since $\mathrm{NMN}^{+}$is an analog of $\mathrm{NAD}^{+}$, it is an open question whether $\mathrm{NMN}^{+}$, when it accumulates to a high level, can also interact with the numerous proteins that are modulated by $\mathrm{NAD}^{+}$.

The rich information provided by comparative genomic analysis can greatly aid metabolic engineering efforts. By bioprospecting NadV homologs from evolutionarily diverse organisms, we identified $R$. solanacearum NadV, which outperformed the two NadVs that have been previously reported as efficient $\mathrm{NMN}^{+}$-producing enzymes in E. coli $[13,19]$, when they are compared in the same condition at a bench scale (Fig. 3). Since F. tularensis NadE* also showed promise to produce $\mathrm{NMN}^{+}$efficiently, a similar approach may be taken in the future to bioprospect NadE* homologs. 
To gain fundamental understanding of nicotinamide cofactor biosynthesis, the mass spectrometry method reported in this work needs to be further expanded to quantify the biosynthetic intermediates of $\mathrm{NMN}^{+}$, such as $\mathrm{NR}$ and $\mathrm{NaMN}^{+}$. While we only produced $\mathrm{NMN}^{+}$in oxidized form, quantification of intracellular $\mathrm{NMNH}$ is also important when enzymes that can cycle $\mathrm{NMN}^{+}$are introduced. Our future work will focus on investigating the reduction potential of $\mathrm{NMN}(\mathrm{H})$ redox pair in vivo, as well as its interplay with $\mathrm{NAD}(\mathrm{P}) / \mathrm{H}$ redox pairs.

An intracellular $\mathrm{NMN}^{+}$level of $1.5 \mathrm{mM}$ is comparable to the levels of native cofactors found $E$. coli, and similar concentrations have been shown to enable NMNdependent biotransformation in whole cells [13]. In the future, additional work will be performed to determine the optimal $\mathrm{NMN}^{+}$production levels to pair with cellfree applications. Moving forward, culture medium and growth conditions can be optimized to potentially yield increased intracellular $\mathrm{NMN}^{+}$levels. In this work, all $\mathrm{NMN}^{+}$production was performed with laboratory standard medium and without optimization. Marinescu and coworkers demonstrated a 32.7-fold increase from $0.72 \mathrm{mM}$ to $23.57 \mathrm{mM}$ of intracellular $\mathrm{NMN}^{+}$upon scaleup to a $500 \mathrm{~mL}$ bioreactor while optimizing $\mathrm{pH}$, NA feeding concentration, and dissolved oxygen while culturing in PYA8 medium [19]. Therefore, performing a similar scale-up with our $\mathrm{NMN}^{+}$producing strain may yield significant increases in $\mathrm{NMN}^{+}$production.

In addition, host selection may play a significant role in efficient $\mathrm{NMN}^{+}$biosynthesis. While most industrial model hosts including E. coli and S. cerevisiae utilize a nicotinic acid adenine dinucleotide (NaAD)-mediated route for de novo $\mathrm{NAD}^{+}$biosynthesis, a small group of prokaryotes use $\mathrm{NMN}^{+}$as the primary precursor to $\mathrm{NAD}^{+}[18,24]$. Since $\mathrm{NMN}^{+}$adopts a distinct role and is naturally maintained at a higher level in these organisms [23, 24], the physiological responses to intracellular $\mathrm{NMN}^{+}$accumulation may be different. Thus, organisms which utilize $\mathrm{NMN}^{+}$-mediated $\mathrm{NAD}^{+}$biosynthesis may be interesting targets for metabolic engineering.

Ultimately, efficient and cost-effective production and purification of $\mathrm{NMN}^{+}$is key for the long-term viability of $\mathrm{NMN}^{+}$-based cell-free biotransformation. Once upstream pathways for the renewable production of $\mathrm{NMN}^{+}$are further established, $\mathrm{NMN}^{+}$will need to be extracted and purified before use in cell-free systems. Cells can be isolated through centrifugation, washed, and lysed through homogenization to isolate $\mathrm{NMN}^{+}$from cellular debris. Alternatively, cells can also be permeabilized to release $\mathrm{NMN}^{+}$across the cell membrane, allowing for fewer steps of isolating $\mathrm{NMN}^{+}$from cell mass. Finally, a major advantage of producing $\mathrm{NMN}^{+}$in vivo is the direct compatibility with crude lysate-based cell-free and whole-cell biosynthesis. By using cells that are capable of both producing intracellular $\mathrm{NMN}^{+}$and expressing enzymes of interest, crude lysates or whole cells can be directly used for $\mathrm{NMN}^{+}$-dependent biosynthesis without the exogenous supply of redox cofactors.

\section{Conclusions}

In this work, we explored routes to efficiently produce $\mathrm{NMN}^{+}$in E. coli. After surveying the routes for $\mathrm{NMN}^{+}$ production in vivo, bioprospecting NadVs enabled the production of $1.5 \mathrm{mM}$ of $\mathrm{NMN}^{+}$using the NadV from $R$. solanacearum. Under the conditions tested, $R$. solanacearum outperformed the previous best NadV's shown to accumulate $\mathrm{NMN}^{+}$efficiently [13, 19]. In addition, this work began to elucidate the physiological effects of $\mathrm{NMN}^{+}$accumulation in E. coli. However, further investigation is necessary to maintain productivity as $\mathrm{NMN}^{+}$ levels are further increased. Ultimately, advancing noncanonical redox cofactor biosynthesis in microorganisms may enable the application of self-sustained, fully renewable cell-free and whole-cell biocatalysis.

\section{Methods}

\section{Plasmid and strain construction}

All molecular cloning was performed in E. coli XL1-Blue cells (Stratagene). A summary of strains and plasmids used in this study can be found in Table 1. Plasmids were assembled by Gibson Isothermal DNA Assembly [38]. Polymerase chain reaction (PCR) fragments were generated using PrimeSTAR Max DNA Polymerase (TaKaRa). The method for plasmid construction is described below.

The $y q h D$ gene was isolated from E. coli BL21 chromosomal DNA by PCR. The resulting PCR fragment was gel purified and assembled into a ColE1 ori, AmpR vector backbone by Gibson isothermal DNA assembly method. We used the $y q h D$-harboring plasmid (pWB301) as a control vector in the growth rescue experiments, because it expresses a similar sized-protein to the $\mathrm{NMN}^{+}$-producing enzymes using the same promoter, and hence may cause similar growth burden. The gene product of $y q h D$ has unrelated function to $\mathrm{NMN}^{+}$ biosynthesis.

E. coli pnuC, S. enterica nadR, S. cerevisiae NRK1, Synechocystis sp nadV, and S. elongatus nadV were isolated by PCR from their respective chromosomal DNA. $F$. tularensis nadE*, R. solanacearum nadV, and $H$. ducreyi $n a d V$ genes were amplified from $E$. coli codon optimized synthesized DNA templates and assembled as described above.

S. enterica РnuC* is generated by site-directed mutagenesis based on the wild type $S$. enterica pnuC gene [30]. The S. enterica pnuC gene was isolated by PCR from chromosomal DNA and assembled as discussed above. 
Table 1 Strains and plasmids used in this study

\begin{tabular}{|c|c|c|}
\hline Strains & Description & Reference \\
\hline XL-1 Blue & Cloning strain & Stratagene \\
\hline BW25113 & E. coli $\triangle(\operatorname{araD}$-araB)567, $\triangle$ lacZ4787(::rrnB-3), $\lambda$, rph-1, $\Delta($ rhaD- rhaB) 568, hsdR514 & Invitrogen \\
\hline JW2670-1 & BW25113 $\Delta p n c C:: k a n$ & $\begin{array}{l}\text { Yale E. coli } \\
\text { Genetic Stock } \\
\text { Center }\end{array}$ \\
\hline MX101 & BW25113 $\Delta$ pncC $\Delta$ nadR::kan & {$[13]$} \\
\hline $72 \mathrm{C}$ & E. coli F-, lacZ4, nadD72(ts,Fs), $\lambda^{-}$, argG75 & {$[28]$} \\
\hline Plasmids & Descriptions & Reference \\
\hline pWB203 & 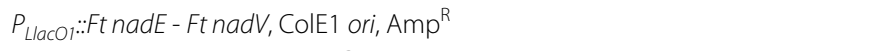 & {$[13]$} \\
\hline pWB301 & 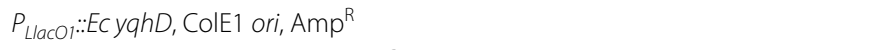 & This study \\
\hline pWB302 & $P_{\text {Llacol }}:$. Se pnuC ${ }^{*} K A$, ColE1 ori, Amp ${ }^{R}$ & This study \\
\hline pWB303 & 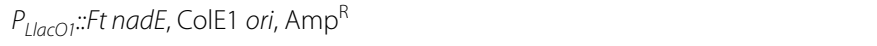 & This study \\
\hline pWB304 & 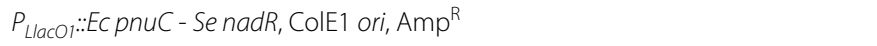 & This study \\
\hline pWB305 & 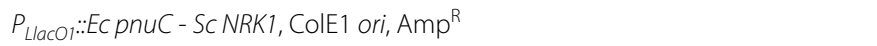 & This study \\
\hline pWB306 & 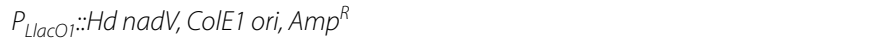 & This study \\
\hline pWB307 & 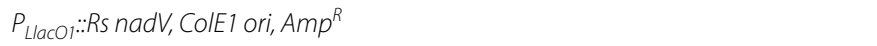 & This study \\
\hline pWB308 & 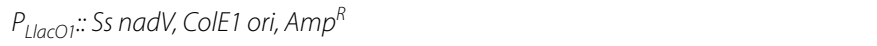 & This study \\
\hline pWB309 & $P_{\text {Llacol }}:$. SynE nadV, ColE1 ori, Amp $p^{R}$ & This study \\
\hline pDB102 & 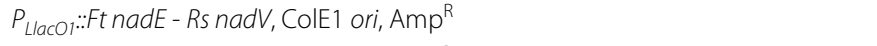 & This study \\
\hline pDB103 & 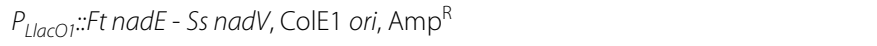 & This study \\
\hline pDB104 & 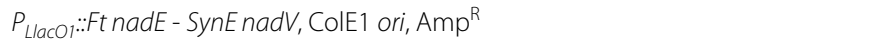 & This study \\
\hline pLZ301 & 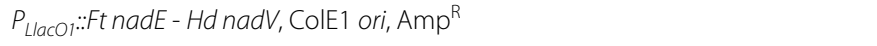 & This study \\
\hline
\end{tabular}

Abbreviations indicate source of genes: Ec Escherichia coli, Se Salmonella enterica, Ft Francisella tularensis, Sc Saccharomyces cerevisiae, Rs Ralstonia solanacearum, Ss Synechocystis sp. PCC 6803, SynE Synechococcus elongatus PCC 7942, Hd Haemophilus ducreyi. Se pnuC* KA contains mutations compared to the wild type sequence (see "Methods" and Additional file 1)

To perform the KA insertion, which has been shown to enable $\mathrm{NMN}^{+}$transport [30], a sequence of AAAGCA was inserted directly after the 321 base pair, as shown in red text in the Supplemental Information. This resulted in the insertion of a lysine and an alanine residue at the 108 and 109 residue positions. The insertion was introduced by PCR. The subsequent mutant PCR fragments were assembled at discussed above. To generate multigene plasmids, the genes were inserted sequentially with a ribosome binding site preceding each gene in the synthetic operon.

Gene sequences are listed in the Additional file.

\section{Growth-based screening platform}

Plasmids (selected from pWB301-305) were transformed into E. coli strains BW25113 and 72c [28] using the Mix \& Go E. coli Transformation Kit (Zymo Research).

Overnight cultures were grown in LB medium supplemented with $2 \mathrm{~g} / \mathrm{L}$ D-glucose, $0.1 \mathrm{mM}$ IPTG, appropriate antibiotics in test tubes at $30{ }^{\circ} \mathrm{C}$ while shaking at 250 r.p.m. for 16 h. For the growth assay, cells were cultured in $1 \mathrm{~mL}$ of LB medium supplemented with $2 \mathrm{~g} / \mathrm{L}$ $D$-glucose, $0.1 \mathrm{mM}$ isopropyl- $\beta$-D-thiogalactopyranoside (IPTG), appropriate antibiotics, and $200 \mu \mathrm{M}$ of feeding compound, if applicable, in a $2 \mathrm{~mL}$ deep-well plate, with square wells, sealed with air permeable film. The medium was inoculated with $1 \% \mathrm{v} / \mathrm{v}$ overnight cultures. Cultures were grown at $42{ }^{\circ} \mathrm{C}$ while shaking at 250 r.p.m.. Cell growth was monitored by measuring optical density at $600 \mathrm{~nm}$. When applicable, media contained $100 \mathrm{mg} / \mathrm{L}$ ampicillin to maintain the plasmids.

\section{Intracellular $\mathrm{NMN}^{+}$generation and quantification}

$\mathrm{NMN}^{+}$generation and quantification were performed as previously reported [13]. Briefly, plasmids (selected from pWB203, pDB102-104, and pLZ301) were transformed into E. coli strains BW25113 and JW2670-1 $(\Delta p n c C)$ as described previously. Overnight cultures were grown in 2xYT medium containing 0.1 mM IPTG, 2 g/L D-glucose, and appropriate antibiotics for $12 \mathrm{~h}$ at $30{ }^{\circ} \mathrm{C}$ at 250 rotations per minute (r.p.m.). To cultivate cells for nucleotide analysis, cells were grown in a $50 \mathrm{~mL}$ conical tube containing $10 \mathrm{~mL}$ of $2 \mathrm{xYT}$ media supplemented with $0.5 \mathrm{mM}$ IPTG, $1 \mathrm{mM}$ nicotinamide, and appropriate antibiotics. Cultures were inoculated with $1 \% \mathrm{v} / \mathrm{v}$ overnight culture. Tubes were incubated at $30{ }^{\circ} \mathrm{C}$ with shaking at 250 r.p.m. for $4 \mathrm{~h}$. All media contained $100 \mathrm{mg} / \mathrm{L}$ ampicillin to maintain the plasmids. 
Cells were processed as previously reported [13]. Briefly, $1 \mathrm{~mL}$ of cell culture was pelleted, washed with $1 \mathrm{~mL}$ of deionized water, and lysed by resuspension in $1 \mathrm{~mL}$ of $95^{\circ} \mathrm{C} 1 \%$ formic acid containing $1 \mu \mathrm{M}$ of 1 -methylnicotinamide as an internal standard. Lysates were quenched in an ice bath before pelleting cell debris. Supernatants were run on a Waters ACQUITY Ultra Performance Liquid Chromatograph with a Waters ACQUITY UPLC CSH C18 column $(1.7 \mu \mathrm{M} \times 2.1 \mathrm{~mm}$ x $50 \mathrm{~mm}$ ). Mobile phases used for separation were (A) water with $2 \%$ acetonitrile and $0.2 \%$ acetic acid and (B) acetonitrile with $0.2 \%$ acetic acid. MS/MS detection was performed by a Waters Micromass Quattro Premier XE Mass Spectrometer. The detailed UPLC and MS parameters were reported previously [13]. Values from LC-MS/ MS were correlated back to intracellular concentration using the number of cells per unit $\mathrm{OD}_{600 \mathrm{~nm}}$ in $1 \mathrm{~mL}$ of culture $=1 \times 10^{9}$ and the intracellular volume of an E. coli cell as $1 \times 10^{-15} \mathrm{~L}$ per cell [39].

\section{Cell growth with high concentrations of $\mathrm{NMN}^{+}$}

A plasmid containing the $\mathrm{NMN}^{+}$transporter S. enterica PnuC* (pWB302) was transformed into $E$. coli strains JW2670-1 $(\Delta p n c C)$ and MX101 ( $\Delta p n c C \Delta n a d R)$. Overnight cultures were grown in $2 \mathrm{xYT}$ medium supplemented with $1 \mathrm{~g} / \mathrm{L}$ D-glucose, $0.1 \mathrm{mM} \mathrm{IPTG}$, and $100 \mathrm{mg} / \mathrm{L}$ ampicillin at $30^{\circ} \mathrm{C}$ while shaking at 250 r.p.m. for $\sim 14 \mathrm{~h} .1 \mathrm{~mL}$ of overnight culture was pelleted in a $1.5 \mathrm{~mL}$ microcentrifuge tube and washed twice with $1 \mathrm{~mL}$ of $1 \times \mathrm{M} 9$ salts. The washed cells were resuspended in $1 \mathrm{~mL}$ of $1 \mathrm{x}$ M9 salts and used for inoculations. For the growth assay, cells were cultured in $1 \mathrm{~mL}$ of M9 minimal medium (1x M9 salts, $1 \mathrm{~g} / \mathrm{L}$ glucose, $1 \mathrm{mM} \mathrm{MgSO}$, $0.1 \mathrm{mM} \mathrm{CaCl} 2,40 \mathrm{mg} / \mathrm{L} \mathrm{FeSO}_{4}$, $1 x$ A5 trace metals with cobalt) containing $0.5 \mathrm{mM}$ IPTG in a $2 \mathrm{~mL}$ deep-well plate, with square wells, sealed with air permeable film. The medium was inoculated with $1 \% \mathrm{v} / \mathrm{v}$ of washed overnight cultures. Cultures were grown at $30{ }^{\circ} \mathrm{C}$ while shaking at 250 r.p.m.. Cell growth was monitored by measuring optical density at $600 \mathrm{~nm}$. Media contained $100 \mathrm{mg} / \mathrm{L}$ ampicillin to maintain the plasmid.

\section{Supplementary information}

Supplementary information accompanies this paper at https://doi. org/10.1186/s12934-020-01415-z.

Additional file 1: Figure S1. Francisella tularensis NadE*-based Growth Restoration is Not Nicotinamide Feeding Dependent. A growth restoration platform was used to screen pathways for the efficient generation of nicotinamide mononucleotide $\left(\mathrm{NMN}^{+}\right)$. The Escherichia coli strain 72c [1], note this reference number is for additional files only] contains a temperature sensitive allele of nadD (ts nadD). As a result, this strain cannot grow at $42^{\circ} \mathrm{C}$. By overexpressing Francisella tularensis nadE*, cells are able to produce $\mathrm{NMN}^{+}$, which can then be converted to $\mathrm{NAD}^{+}$, and thus restoring growth. We observed no dependence of growth restoration with feeding $200 \mu \mathrm{M}$ nicotinamide (NA). This indicates either efficient $\mathrm{NMN}^{+}$generation can be achieved through channeling the intermediate nicotinic acid mononucleotide $\left(\mathrm{NaMN}^{+}\right)$from $E$. coli's native de novo $\mathrm{NAD}^{+}$biosynthesis pathway, or LB medium used in this experiment already contains sufficient precursors for this pathway. Screening was performed in a deep-well 96-well plate containing $1 \mathrm{~mL}$ of LB medium supplemented with $2 \mathrm{~g} / \mathrm{L}$ D-glucose and $200 \mu \mathrm{M}$ of NA if applicable. Detailed conditions are described in the Methods section. Figure S2. Francisella tularensis $\mathrm{NadE}^{*}$ Expression Alleviates $R$. solanacearum NadV Growth Challenge. BW25113 $\triangle p n c C$ cells expressing R. solanacearum NadV demonstrate a growth challenge. However, when paired with F. tularensis NadE*, the growth challenge is alleviated. Interestingly, this challenge is not seen when H. ducreyi NadV is expressed. Therefore, F. tularensis $\mathrm{NadE}^{*}$ may play a synergistic role in stability, activity, or expression of some NadV candidates. Cells were cultured identically to the intracellular $\mathrm{NMN}^{+}$generation cultures described in the Methods section. Cell growth was monitored by measuring optical density at $600 \mathrm{~nm}$. Abbreviations indicate source of genes: Ft, Francisella tularensis, Hd, Haemophilus ducreyi; Rs, Ralstonia solanacearum. Figure $\mathbf{S 3}$. Intracellular $\mathrm{NAD}^{+}$Decreases in $\mathrm{NMN}^{+}$Accumulating Strains From Fig. 3, co-overexpression of $\mathrm{NMN}^{+}$ generating Francisella tularensis NadE* and NadVs increases intracellular $\mathrm{NMN}^{+}$when the $\mathrm{NMN}^{+}$degrading PncC is disrupted. However, as shown here, $\mathrm{NAD}^{+}$levels decreased in cells expressing F. tularensis $\mathrm{NadE}^{*}$ and NadV compared to cells without overexpression. This potentially indicates $\mathrm{NMN}^{+}$plays a regulatory role in $\mathrm{NAD}^{+}$biosynthesis. Cells were cultured in 2XYT medium supplemented with $1 \mathrm{mM}$ nicotinamide at $30^{\circ} \mathrm{C}$ for $4 \mathrm{~h}$. Intracellular NAD ${ }^{+}$concentrations were determined by UPLC-MS/ MS. Detailed conditions and analytical techniques are described in the Methods section.DNA Sequences of Genes Used in This Study: List of DNA sequences of the genes used in this study.

\section{Abbreviations}

$\mathrm{NAD}^{+}$: Nicotinamide adenine dinucleotide; NADP+: Nicotinamide adenine dinucleotide phosphate; P2NA ${ }^{+}$: 3-carbomoyl-1-phenethylpyridin-1-ium chloride; $\mathrm{NMN}^{+}$: Nicotinamide mononucleotide; NadV: Nicotinamide phosphoribosyltransferases; NadE*: Nicotinamide mononucleotide synthase; PncC: Nicotinamide mononucleotide; $\mathrm{NaMN}^{+}$: Nicotinic acid mononucleotide; NR: Nicotinamide riboside; PnuC: Nicotinamide riboside transporter; PnuC*: Mutant nicotinamide riboside transporter; Nrk1: Nicotinamide riboside kinase from Saccharomyces cerevisiae; NadR: Nicotinamide riboside kinase (Salmonella enterica); NA: Nicotinamide; LC-MS: Liquid chromatography-mass spectrometry; NaAD: Nicotinic acid adenine dinucleotide; PCR: Polymerase chain reaction; IPTG: Isopropyl- $\beta$-D-thiogalactopyranoside; r.p.m.: Rotations per minute.

\section{Acknowledgements}

We thank the University of California, Irvine Mass Spectrometry Facility and Dr. Felix Grun for help with LC-MS.

\section{Authors' contributions}

HL conceived the research. WBB, LZ, EK designed and conducted growthbased screening. WBB, DB, DA performed study of prospective NadV homologs. WBB designed and WBB, DA, DB, LZ, EK, performed the intracellular $\mathrm{NMN}^{+}$and $\mathrm{NAD}^{+}$level analysis. WBB and DA investigated physiological response to intracellular $\mathrm{NMN}^{+}$. All authors analyzed the data and interpreted results. HL, WBB, DA wrote the manuscript. All authors read and approved the final manuscript.

\section{Funding}

H.L. acknowledges support from University of California, Irvine, the National Science Foundation (NSF) (award no. 1847705), and the National Institutes of Health (NIH) (award no. DP2 GM137427). W.B.B. acknowledges support from Graduate Assistance in Areas of National Need fellowship funded by the U.S. Department of Education. D.A. acknowledges support from the Federal Work Study Program funded by the U.S. Department of Education. The content is solely the responsibility of the authors and does not necessarily represent the official views of the National Institutes of Health or the NSF. 


\section{Availability of data and materials}

The datasets used and/or analyzed during this study are available from the corresponding author on reasonable request.

\section{Competing interests}

The authors declare no competing financial interests.

\section{Author details}

${ }^{1}$ Departments of Chemical and Biomolecular Engineering, University of California, Irvine, CA, United States. ${ }^{2}$ Molecular Biology and Biochemistry, University of California, Irvine, CA, United States.

Received: 12 May 2020 Accepted: 20 July 2020

Published online: 27 July 2020

\section{References}

1. Bowie JU, Sherkhanov S, Korman TP, Valliere MA, Opgenorth PH, Liu H. Synthetic Biochemistry: The Bio-inspired Cell-Free Approach to Commodity Chemical Production. Trends Biotechnol. 2020. in press.

2. Dudley QM, Karim AS, Jewett MC. Cell-free metabolic engineering: biomanufacturing beyond the cell. Biotechnol J. 2015;10(1):69-82.

3. Wilding KM, Schinn S, Long EA, Bundy BC. The emerging impact of cellfree chemical biosynthesis. Curr. Opin. Biotech. 2018;53:115-21.

4. Honda K, Kimura K, Ninh PH, Taniguchi H, Okano K, Ohtake H. In vitro bioconversion of chitin to pyruvate with thermophilic enzymes. J Biosci Bioeng. 2017;124(3):297-301.

5. Wang W, Liu M, You C, Li Z, Zhang YHP. ATP-Free biosynthesis of a high-energy phosphate metabolite fructose 1,6-diphosphate by in vitro metabolic engineering. Metab Eng. 2017:42:168-74.

6. Kay JE, Jewett MC. A cell-free system for production of 2,3-butanediol is robust to growth-toxic compounds. Metab Eng. Commun. 2020;10:e00114.

7. Karim AS, Jewett MC. A cell-free framework for rapid biosynthetic pathway prototyping and enzyme discovery. Metab Eng. 2016;36:116-26.

8. Kelwick R, Ricci L, Chee SM, Bell D, Webb AJ, Freemont PS. Cell-free prototyping strategies for enhancing the sustainable production of polyhydoxyalkanoates bioplastics. Synth Bio. 2018;3(1):1-12.

9. Nowak C, Pick A, Csepei L, Sieber V. Characterization of biomimetic cofactors according to stability, redox potentials, and enzymatic conversion by NADH oxidase from Lactobacillus pentosus. ChemBioChem. 2017;18(19):1944-9.

10. Nowak $C$, Pick A, Lommes P, Sieber $V$. Enzymatic reduction of nicotinamide biomimetic cofactors using an engineered glucose dehydrogenase: providing a regeneration system for artificial cofactors. ACS Catal. 2017;7(8):5202-8.

11. Kaplan NO, Ciotti MM, Stolzenbach FE. Reaction of pyridine nucleotide analogues with dehydrogenases. J Biol Chem. 1956;221:833-44.

12. Kaufman RA. Use of Nicotinamide Adenine Dinucleotide (NAD) Analogs to Measure Ethanol. European Patent Specification Patent No. EP 1242 440 B1, filed December 27, 2000, and published September 16, 2015.

13. Black WB, Zhang L, Mak WS, Maxel S, Cui Y, King E, Fong B, Sanchez Martinez A, Siegel JB, Li H. Engineering a nicotinamide mononucleotide redox cofactor system for biocatalysis. Nat Chem Biol. 2020;16(1):87-94.

14. Campbell E, Meredith M, Minteer SD, Banta S. Enzymatic biofuel cells utilizing a biomimetic cofactor. Chem Commun. 2012;48:1898-900.

15. Ji DB, Wang L, Liu W, Hou S, Zhao KZ. Synthesis of NAD analogs to develop bioorthogonal redox system. Sci China Chem. 2013;56(3):296-300

16. Paul CE, Arends IWCE, Hollmann F. Is simpler better? synthetic nicotinamide cofactor analogues for redox chemistry. ACS Catal. 2014;4(3):788-97.

17. Makarov MV, Migaud ME. Syntheses and chemical properties of $\beta$-nicotinamide riboside and its analogues and derivatives. Beilstein J Org Chem. 2019;15:401-30.

18. Gazzaniga F, Stebbins R, Chang SZ, McPeek MA, Brenner C. Microbial NAD metabolism: lessons from comparative genomics. Microbiol Mol Bio Rev. 2009;73(3):529-41.

19. Marinescu GC, Popescu R, Stoian G, Dinischiotu A. ß-nicotinamide mononucleotide (NMN) production in Escherichia coli. Sci Rep. 2018;8:12278.
20. Sinclair DA, EAR PH. Biological Production of NAD Precursors and Analogs. WO Patent WO 2015/069860 Al, filed November 6, 2014, and issued, May 14,2015

21. Paul CE, Gargiulo S, Opperman DJ, Lavandera I, Gotor-Fernández V, Gotor $\checkmark$, Taglieber A, Arends IWCE, Hollmann F. Mimicking nature: synthetic nicotinamide cofactors for $\mathrm{C}=\mathrm{C}$ bioreduction using enoate reductases. Org Lett. 2013;15(1):180-3.

22. Rollin JA, Tam TK, Zhang YHP. New biotechnology paradigm: cell-free biosystems for biomanufacturing. Green Chem. 2013;15:1708-19.

23. Evans C, Bogan KL, Song P, Burant CF, Kennedy RT, Brenner C. NAD ${ }^{+}$ metabolite levels as a function of vitamins and calorie restriction: evidence for different mechanisms of longevity. BMC Chem Biol. 2010;10(2):1-10.

24. Sorci L, Martynowski D, Rodionov DA, Eyobo Y, Zogaj X, Klose KE, Nikolaev EV, Magni G, Zhang H, Osterman AL. Nicotinamide mononucleotide synthetase is the key enzyme for an alternative route of NAD biosynthesis in Francisella tularensis. PNAS. 2009;106(9):3083-8.

25. Sauer E, Merdanovic M, Mortimer AP, Bringmann G, Reidl J. PnuC and the utilization of the nicotinamide riboside analog 3-aminopyridine in haemophilus influenzae. Antimicrob Agents Chemother. 2004:48(12):4532-41.

26. Belenky P, Christensen KC, Gazzaniga F, Pletnev AA, Brenner C. Nicotinamide riboside and nicotinic acid riboside salvage in fungi and mammals. J Biol Chem. 2009;284(1):158-64.

27. Kurnasov OV, Polanuyer BM, Ananta S, Sloutsky R, Tam A, Gerdes S, Osterman A. Ribosylnicotinamide kinase domain of NadR protein: identification and implications in NAD biosynthesis. J Bacteriol. 2002;184(24):6906-17.

28. Stancek $M$, Isaksson LA, Rydén-Aulin M. fusB is an allele of nadD, encoding nicotinate mononucleotide andeylyltransferase in Escherichia coli. Microbiology. 2003;149(9):2427-33.

29. Wang X, Zhou YJ, Wang L, Liu W, Liu Y, Peng C, Zhao ZK. Engineering Escherichia coli nicotinic acid mononucleotide adenylyltransferase for fully active amidated NAD biosynthesis. Appl Environ Microbiol. 2017;83(13):e00692-17

30. Grose JH, Bergthorsson U, Xu Y, Sterneckert J, Khodaverdian B, Roth JR. Assimilation of nicotinamide mononucleotide requires periplasmic AphA phosphatase in Salmonella enterica. J Bacteriol. 2005;187(13):4521-30.

31. Raffaelli NL, Lorenzi T, Mariani PL, Emanuelli M, Amici A, Ruggieri S, Magni G. The Escherichia coli NadR regulator is endowed with nicotinamide mononucleotide adenylyltransferase activity. J Bacteriol. 1999;181(17):5509-11.

32. Tritz GJ, Chandler JL. Recognition of a gene involved in the regulation of nicotinamide adenine dinucleotide biosynthesis. J Bacteriol. 1973;114(1):128-36

33. Zhu NO, Olivera BM, Roth JR. Activity of the nicotinamide mononucleotide transport system is regulated in Salmonella typhimurium. J Bacteriol. 1991;173(3):1311-20.

34. Euro L, Belevich G, Bloch DA, Verkhovsky MI, Wikström M, Verkhovskaya M. The Role of the Invariant Glutamate 95 in the Catalytic Site of Complex I from Escherichia coli. Biochim. Biophys. Acta, Bioenerg. 2009;1787(1):68-73.

35. Leonardo MR, Dailly Y, Clark DP. Role of NAD in regulating the adhE gene of Escherichia coli. J Bacteriol. 1996;178(20):6013-8.

36. Rodionov DA, De Ingeniis J, Mancini C, Cimadamore F, Zhang H, Osterman AL, Raffaelli N. Transcriptional regulation of NAD metabolism in bacteria: nrtR family of Nudix-related regulators. Nucleic Acids Res. 2008;36(6):2047-59.

37. Shone CC, Fromm HJ. Steady-state and pre-steady-state kinetics of coenzyme a linked aldehyde dehydrogenase from Escherichia coli. Biochemistry. 1981;20(26):7494-501.

38. Gibson DG, Young L, Chuang RY, Venter JC, Hutchison CA III, Smith HO. Enzymatic assembly of DNA molecules up to several hundred kilobases. Nat Methods. 2009;6(5):343-5.

39. Findik BT, Randall LL. Determination of the intracellular concnetraiton of the export chaperone SecB in Escherichia coli. PLoS ONE. 2017;12(8):e0183231.

\section{Publisher's Note}

Springer Nature remains neutral with regard to jurisdictional claims in published maps and institutional affiliations. 